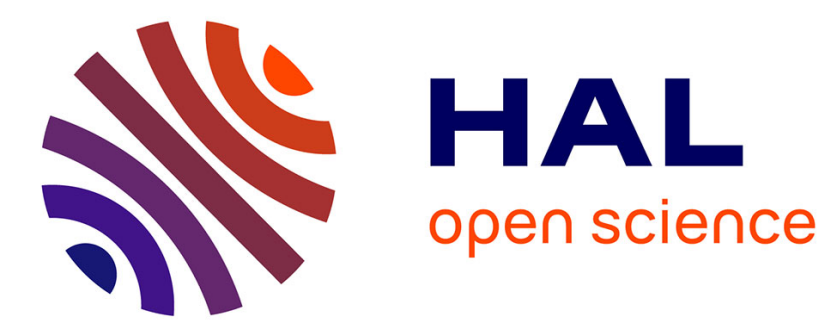

\title{
An Analytical Formulation for the Geometrico-static Problem of Continuum Planar Parallel Robots
}

Federico Zaccaria, Sébastien Briot, Mohamed Taha Chikhaoui, Edoardo Idá, Marco Carricato

\section{- To cite this version:}

Federico Zaccaria, Sébastien Briot, Mohamed Taha Chikhaoui, Edoardo Idá, Marco Carricato. An Analytical Formulation for the Geometrico-static Problem of Continuum Planar Parallel Robots. 23rd CISM IFToMM Symposium on Robot Design, Dynamics and Control (RoManSy 2020), Sep 2020, Sapporo, Japan. hal-02849910

\section{HAL Id: hal-02849910 https://hal.science/hal-02849910}

Submitted on 22 Jun 2020

HAL is a multi-disciplinary open access archive for the deposit and dissemination of scientific research documents, whether they are published or not. The documents may come from teaching and research institutions in France or abroad, or from public or private research centers.
L'archive ouverte pluridisciplinaire HAL, est destinée au dépôt et à la diffusion de documents scientifiques de niveau recherche, publiés ou non, émanant des établissements d'enseignement et de recherche français ou étrangers, des laboratoires publics ou privés. 


\title{
An Analytical Formulation for the Geometrico-static Problem of Continuum Planar Parallel Robots
}

\author{
Federico Zaccaria ${ }^{1}$, Sébastien Briot ${ }^{2}$, M. Taha Chikhaoui ${ }^{3}$, \\ Edoardo Idà ${ }^{1}$, and Marco Carricato ${ }^{1}$ \\ ${ }^{1}$ DIN, University of Bologna, Bologna, Italy \\ ${ }^{2}$ CNRS, Laboratoire des Sciences du Numérique de Nantes (LS2N), France \\ ${ }^{3}$ CNRS, Laboratoire TIMC-IMAG, Grenoble, France \\ federico.zaccaria2@studio.unibo.it, Sebastien.Briotels2n.fr \\ taha.chikhaoui@univ-grenoble-alpes.fr, \\ \{edoardo.ida2, marco.carricato\}eunibo.it
}

\begin{abstract}
In this paper, we provide an analytical formulation for the geometricostatic problem of continuum planar parallel robots. This formulation provides to an analytical computation of a set of equations governing the equilibrium configurations. We also introduce a stability criterion of the computed configurations. This formulation is based on the use of Kirchhoff's rod deformation theory and finite-difference approximations. Their combination leads to a quadratic expression of the rod's deformation energy. Equilibrium configurations of a planar parallel robot composed of two hinged flexible rods are computed according to this new formulation and compared with the ones obtained with state-of-the-art approaches. By assessing equilibrium stability with the proposed technique, new unstable configurations are determined.
\end{abstract}

\section{Introduction}

The need of developing robots which can safely interact with their environments has lead robotics researchers to design a new type of robot manipulators named continuum robots [7]. Most of these mechanisms feature a serial architecture, in which rigid bodies and joints are replaced by an assembly of slender rods deformed by wires, electromagnets, fluidic actuators or other types of actuation. Continuum robots, inspired by biological systems such as trunks, tentacles, and snakes, have been used in many applications in which the problem of manipulation in confined, or hard-to-reach workspace, is crucial, like minimally invasive surgery $[8,9,11]$.

The concept of parallel continuum robots was introduced in [5,6]. These first works dealt with the design of continuum Gough-Stewart-like platforms. The robot legs were modeled by using the Cosserat rod theory, and the system of nonlinear ordinary differential equations characterizing the robot's equilibrium configurations was solved by a purely numerical approach (shooting method). More recently, several designs of continuum planar parallel robots (CPPRs) have been proposed [1-4], and their geometricostatic problems were analyzed. These mechanisms were modeled by using the Kirchhoff's rod theory, and the authors proposed a quasi-analytical description of the robot equilibrium configurations. This quasi-analytical form was obtained under the strict 
condition that external wrenches can only be applied to the platform, and that the deformations are planar. Wrenches applied to other bodies cannot be handled, and spatial robots cannot be modeled. Additionally, according to [1-5], stability analysis of equilibrium configurations is not straightforward. Stability can be assessed according to the numerical method introduced in [12].

In this paper, an alternative formulation stemming from a simpler mathematical framework is introduced. Our approach allows analytical computation of the manipulator's total potential energy and of its geometrical constraint equations. The minimization of the potential energy subject to geometrical constraints allows for the computation of robot equilibrium configurations. Furthermore, equilibrium stability can be assessed by computing the Hessian matrix of a Lagrangian function [10].

The paper is structured as follows. Section 2 deals with the analytical formulation of the geometrico-static problems and with the computation of the Lagrange function Hessian matrix for generic CPPRs. Section 3 focuses on the geometrico-static analysis of a $C P P R$ composed of two hinged flexible rods. Comparison of results obtained with our approach and with the shooting method are provided. The stability analysis, based on the positiveness of the Hessian matrix, is checked on known stable and unstable equilibria. Further, previously undetected unstable configurations are determined. In the last Section, conclusions are drawn.

\section{Geometrico-static Modelling of CPPRs}

\subsection{Deformation energy of a planar slender rod}

Let us consider an undeformed straight slender rod (Fig. 1(a)), made of an isotropic material and having a constant cross-section along its longitudinal direction. By using the Kirchhoff's modeling assumption (shear and extensibility of the beam are neglected), the deformation energy, when considering only deformations in the plane $(O x y)$, is given by:

$$
V_{e}=\frac{1}{2} \int_{0}^{L} E I_{z z} \theta^{\prime 2} d s
$$

where $E$ is the material Young's modulus, $I_{z z}$ is the area moment of inertia of the cross-section, $\theta^{\prime}=d \theta / d s$ is the derivative of the rotation $\theta$ of the rod cross-section (see Fig. 1(a)), $L$ is the rod length, and $s$ is the curvilinear abscissa along the rod.

It is possible to approximate the expression of $\theta^{\prime}$ by using finite differences [11]. For this, let us discretize the rod into $N$ elements of equal length $\ell_{e}=L / N$ (Fig. 1(b)). The expression of the deformation energy thus becomes:

$$
V_{e} \approx \sum_{i=1}^{N} V_{e i}, \text { where } V_{e i}=\frac{1}{2} \int_{0}^{\ell_{e}} E I_{z z} \theta_{i}^{\prime 2} d s
$$

with $\theta_{i}^{\prime}$ being the derivative of the rotation $\theta_{i}$ of the element $i$ (Fig. 1(b)). The expression of $\theta_{i}^{\prime}$ can be approximated by using finite differences as follows:

$$
\theta_{i}^{\prime}=\frac{\theta_{i}-\theta_{i-1}}{\ell_{e}},
$$






(a) Continuous parameterization

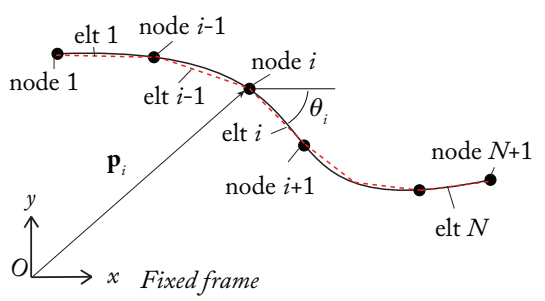

(b) Discrete parameterization

Fig. 1. Parameterization of the deformation of a slender rod

with $\theta_{1}=\theta_{0}$ if the rod is assumed to be clamped at its base $(s=0)$.

Then, introducing Eq. (3) into Eq. (2) leads to:

$$
V_{e i}=\frac{1}{2} \frac{E I_{z z}}{\ell_{e}}\left(\theta_{i}-\theta_{i-1}\right)^{2}
$$

From Eq. (4), we can notice that the deformation energy is analogous to the energy of a torsional spring of stiffness $K_{e q}=E I_{z z} / \ell_{e}$ located between elements $i-1$ and $i$.

Now, considering external constant force $\mathbf{f}_{i}$ and moment $m_{i}$ applied at node $i^{4}$, the potential energy associated to this loading is equal to:

$$
V_{l i}=-\mathbf{f}_{i}^{T}\left(\mathbf{p}_{i}-\mathbf{p}_{i}^{0}\right)-m_{i}\left(\theta_{i}-\theta_{i}^{0}\right)=-\left[\left(\mathbf{p}_{i}-\mathbf{p}_{i}^{0}\right)^{T} \theta_{i}-\theta_{i}^{0}\right]^{T} \mathbf{w}_{i}
$$

where $(.)^{0}$ denotes the undeformed state [11], $\mathbf{w}_{i}=\left[\mathbf{f}_{i}^{T} m_{i}\right]^{T}$ is the external loading, and $\mathbf{p}_{i}$ is the position of node $i$, which can be computed by the recursive formula:

$$
\mathbf{p}_{i}=\mathbf{p}_{i-1}+\ell_{e}\left[\begin{array}{c}
\cos \theta_{i} \\
\sin \theta_{i}
\end{array}\right], \text { for } i=2, \ldots, N
$$

with $\mathbf{p}_{1}$ being a known position if the rod is clamped at $s=0$.

Thus, the total potential energy of the rod, function of rotations $\theta_{1}$ to $\theta_{N}$, is:

$$
V_{\text {rod }}\left(\theta_{1}, \ldots, \theta_{N}\right)=\sum_{i=1}^{N}\left(V_{e i}+V_{l i}\right)+V_{l,(N+1)}
$$

\subsection{Potential energy of CPPRs}

Let us consider a CPPR composed of $n$ continuum chains (called legs) (Fig. 2(a)). In this paper, legs will be considered as identical, actuated by a revolute motor at one end (points $A_{k}, k=1, \ldots, n$ ) and attached to a rigid platform via passive revolute joints at the other end (points $\left.B_{k}, k=1, \ldots, n\right)^{5}$.

\footnotetext{
${ }^{4}$ Gravitational loads were neglected for brevity sake, but they can be introduced into Eq. (5) as nodal external actions $\mathbf{f}_{i}$ and $m_{i}$

${ }^{5}$ Other types of joints could be considered as well, but we focus on revolute joints for the sake of brevity.
} 


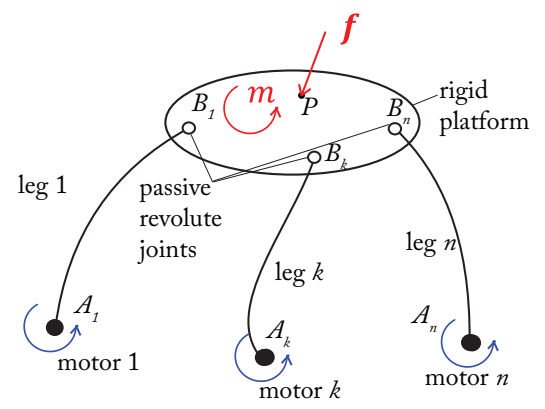

(a) A general $C P P R$



(b) $C P P R$ made of 2 slender rods

Fig. 2. Schematics of $C P P R$

The total potential energy of the $C P P R$ is given by:

$$
V_{t o t}\left(\mathbf{q}_{a}, \boldsymbol{\theta}, \mathbf{p}, \phi\right)=\sum_{k=1}^{n} V_{\operatorname{rod}_{k}}\left(q_{a k}, \boldsymbol{\theta}_{k}, \mathbf{w}_{k}\right)-\mathbf{f}^{T}\left(\mathbf{p}-\mathbf{p}^{0}\right)-m\left(\phi-\phi^{0}\right)
$$

where $V_{\text {rod }_{k}}$ is the total potential energy of the leg $k$, which is a function of the rotations $\boldsymbol{\theta}_{k}=\left[\begin{array}{lll}\theta_{k 2} & \ldots & \theta_{k N_{k}}\end{array}\right]^{T}\left(\theta_{k j}\right.$ being the deformation of the element $j$ for leg $k$, and $N_{k}$ being the number of elements for the same leg), of the motor variables $\mathbf{q}_{a}=\left[q_{a 1} \ldots q_{a n}\right]^{T}$ $\left(q_{a 1}=\theta_{k 1}\right)$, and of the nodal loadings $\mathbf{w}_{k}=\left[\mathbf{w}_{k 1}^{T} \ldots \mathbf{w}_{k N}^{T}\right]\left(\mathbf{w}_{k i}\right.$ is the nodal wrench on the node $i$ of the leg $k$ ), $\mathbf{f}$ is a constant force exerted on the robot platform, $m$ is a constant moment, $\mathbf{p}$ is the location of the force application point $P$, and $\phi$ is the platform's orientation (Fig. 2(a)). Moreover, $\boldsymbol{\theta}=\left[\boldsymbol{\theta}_{1}^{T}, \ldots, \boldsymbol{\theta}_{n}^{T}\right]^{T}$.

Variables $\mathbf{q}_{a}, \boldsymbol{\theta}, \phi$ and $\mathbf{p}$ are related by the following geometric constraints:

$$
\boldsymbol{\Phi}_{k}=\mathbf{p}_{B_{k}}(\mathbf{p}, \phi)-\mathbf{p}_{B_{k}}\left(q_{a k}, \boldsymbol{\theta}_{k}\right)=\mathbf{0}
$$

where $\mathbf{p}_{B_{k}}$ is the location of the center of the platform's revolute joint for the leg $k$, and

$$
\mathbf{p}_{B_{k}}(\mathbf{p}, \phi)=\mathbf{p}+\mathbf{R}(\phi, z) \mathbf{p}_{P B_{k}},
$$

where $\mathbf{R}(\phi, z)$ is the (planar) rotation matrix of angle $\phi$ around the $z$-axis and $\mathbf{p}_{P B_{k}}$ the constant vector between points $P$ and $B_{k}$ in the platform frame, and $\mathbf{p}_{B_{k}}\left(\boldsymbol{\theta}_{k}\right)$ can be computed from (6), starting from the known rod extremity located at point $A_{k}$. In what follows, we denote as $\boldsymbol{\Phi}$ the vector stacking all constraints $\boldsymbol{\Phi}_{k}$.

\subsection{Geometrico-static model for CPPRs}

Feasible robot configurations $\mathbf{x}=\left[\boldsymbol{\theta}^{T} \mathbf{p}^{T} \phi\right]^{T}$ are stable static equilibria for fixed motor positions $\mathbf{q}_{a}$. They appears if and only if the robot internal configuration $\mathbf{x}$ leads to a minimum of the potential energy $V_{t o t}$. Lagrange conditions provide the following characterization of local extrema. Under the condition that $\nabla_{\mathbf{x}} \boldsymbol{\Phi}\left(\mathbf{q}_{a}, \mathbf{x}\right)$ is full rank, $\mathbf{x}$ is a local extremum for a fixed motor position $\mathbf{q}_{a}$ if and only if there exist multipliers $\lambda \in \mathbb{R}^{n_{\Phi}}$ ( $n_{\Phi}$ being the length of the constraint vector $\boldsymbol{\Phi}$ ) such that

$$
\nabla_{\mathbf{x}} V_{t o t}\left(\mathbf{q}_{a}, \mathbf{x}\right)+\nabla_{\mathbf{x}} \boldsymbol{\Phi}\left(\mathbf{q}_{a}, \mathbf{x}\right) \boldsymbol{\lambda}=\mathbf{0}, \quad \boldsymbol{\Phi}\left(\mathbf{q}_{a}, \mathbf{x}\right)=\mathbf{0} .
$$


Note that the expression at the left-hand side of Eq. (11) is the gradient $\nabla_{\mathbf{x}} \mathcal{L}\left(\mathbf{q}_{a}, \mathbf{x}\right)$ of the Lagrangian function

$$
\mathcal{L}\left(\mathbf{q}_{a}, \mathbf{x}\right)=V_{\text {tot }}\left(\mathbf{q}_{a}, \mathbf{x}\right)+\boldsymbol{\Phi}\left(\mathbf{q}_{a}, \mathbf{x}\right)^{T} \boldsymbol{\lambda}
$$

The implicit geometrico-static model (11) is a system of $m$ equations ( $m=m_{x}+n_{\Phi}$, where $m_{x}$ is the dimension of $\mathbf{x}$ ) and $m+n$ unknowns. As a consequence, fixing $n$ variables to desired values yields a square system, which generically has a finite number of solutions. The forward geometrico-static problem (FGSP) consists in fixing the $n$ motor positions $\mathbf{q}_{a}$ and computing $n$ controlled coordinates, the uncontrolled coordinates $\mathbf{x}$ and the Lagrange multipliers $\boldsymbol{\lambda}$ so that $\left(\mathbf{q}_{a}, \mathbf{x}, \boldsymbol{\lambda}\right)=\left(\mathbf{q}_{a}, \boldsymbol{\theta}, \mathbf{p}, \phi, \boldsymbol{\lambda}\right)$ is solution to Eq. (11). The inverse geometrico-static problem (IGSP) consists in assigning the $n$ controlled coordinates ( $\mathbf{p}, \phi$ if $n=3$, a subset of it if $n<3$, and $\mathbf{p}, \phi$ and some other variables in $\boldsymbol{\theta}$ if $n>3$ ) and computing the corresponding $n$ motor positions $\mathbf{q}_{a}$, the remaining unknown variables in $\boldsymbol{\theta}$, and the Lagrange multipliers $\boldsymbol{\lambda}$.

\subsection{Equilibrium stability}

At this stage, we do not know if the configuration $\mathbf{q}_{\mathrm{a}}$ and $\mathrm{x}$ solution of the geometricostatic model (11) is stable or not. Without loss of generality, in what follows, we define the stability conditions based on the FGSP equations.

Consider a set of solutions $\left(\mathbf{q}_{a}^{*}, \mathbf{x}^{*}, \boldsymbol{\lambda}^{*}\right)$ to Eqs. (11). The stability of the configuration $\left(\mathbf{q}_{a}^{*}, \mathbf{x}^{*}\right)$ can be checked by verifying the following second-order condition [10]:

$$
\mathbf{H}^{p}\left(\mathbf{q}_{a}^{*}, \mathbf{x}^{*}, \boldsymbol{\lambda}^{*}\right)=\mathbf{Z}^{T} \mathbf{H Z} \succ 0
$$

in which:

- $\mathbf{H}=\nabla_{\mathbf{x}, \mathbf{x}} \mathcal{L}\left(\mathbf{q}_{a}^{*}, \mathbf{x}^{*}, \boldsymbol{\lambda}^{*}\right)$ is the Hessian of the Lagrangian $\mathcal{L}$ (see (12)) with respect to the variables in $\mathbf{x}$ computed for the configuration $\left(\mathbf{q}_{a}^{*}, \mathbf{x}^{*}, \boldsymbol{\lambda}^{*}\right)$

- $\mathbf{Z}$ is the matrix that spans the null space of $\nabla_{\mathbf{x}} \boldsymbol{\Phi}\left(\mathbf{q}_{a}^{*}, \mathbf{x}^{*}\right)$ computed at $\left(\mathbf{q}_{a}^{*}, \mathbf{x}^{*}\right)$, i.e.

$$
\nabla_{\mathbf{x}} \boldsymbol{\Phi}\left(\mathbf{q}_{a}^{*}, \mathbf{x}^{*}\right) \mathbf{Z}=\mathbf{0}
$$

\subsection{Solver}

It is generally impossible to find a closed-form solution for Eq. (11) because of its nonlinearity. Therefore, we use a trust-region algorithm. A multi-start procedure is used in order to identify as many equilibria as possible, i.e. the algorithm is run several times with a random selection of the initial guess.

\section{Case study}

In this Section, we study the CPPR composed of two rods presented in [1] (Fig. 2(b)). It is called a $\underline{R} F R F \underline{R}$ robot because it is composed of two actuated revolute $(\underline{R})$ joints, each being mounted on the ground and attached at one extremity of a flexible rod $(F)$. 


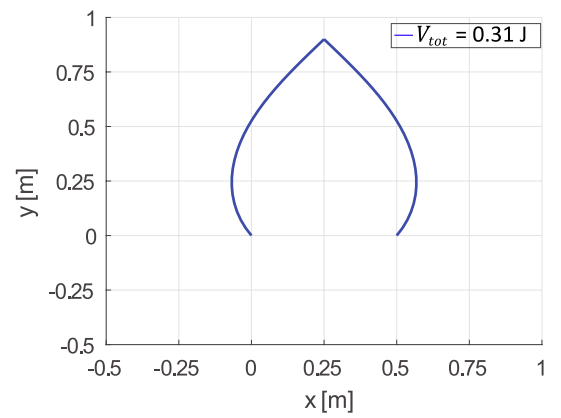

(a) A stable solution



(b) Computation time with respect to the number of elements

Fig. 3. Stable solution and computation time for solving the FGSP, for $q_{a 1}=120 \mathrm{deg}$, $q_{a 2}=60 \mathrm{deg}$

Both flexible rods are connected at their tip through a passive revolute $(R)$ joint. Parameters of the rods are: $L=1 \mathrm{~m}$, circular cross-section of radius $1 \mathrm{~mm}, E=210 \mathrm{GPa}$. The distance $\ell_{A_{1} A_{2}}$ between the two motors is $\ell_{A_{1} A_{2}}=0.5 \mathrm{~m}$.

The aim of this study is not to perform a full geometrico-static evaluation of the robot, but to illustrate the main capabilities of our method, namely the simple equilibrium evaluation and the straightforward stability analysis. First, we compute solutions of the FGSP for $q_{a 1}=120 \mathrm{deg}, q_{a 2}=60 \mathrm{deg}$. We identify a single stable solution (Fig. 3(a)). The evolution of the computational time with respect to the total number of elements for this configuration is provided in Fig. 3(b). Results are provided for a CPU Inter Core i7-6700HQ, $2.60 \mathrm{GHz}, 8 \mathrm{~Gb}$ of RAM. The convergence time becomes high ( $>10 \mathrm{~s}$ ) for a number of elements larger than 100. However, this convergence time is also highly dependent on the initial guess. If the solution is close enough, the algorithm converges in a few iterations.

Accuracy of the computed solutions for both the forward and inverse geometricostatic problems is assessed in Fig. 4. We compare our results with those obtained by the geometrically exact shooting method [5] directly applied to the set of differential equations characterizing the robot. Obviously, the accuracy of our method is directly related to the number of elements that we used. In general, a difference of less than $1 \mathrm{~mm}$ (for a robot composed of two rods of $1 \mathrm{~m}$ ) on the prediction of the location of the end-effector is obtained when the number of elements per leg exceeds 100.

Finally, we check the stability of the computed equilibria based on criterion (13). All equilibria identified as stable in [1-4] (Figs. 3 and 4) lead to the positive-definiteness of the Hessian, whereas the unstable equilibrium shown in [2] (Fig. 5(a)), did not satisfy the condition (13). Based on the criterion (13), we are able to find more unstable configurations (Fig. 5(b)) than those identified before.

These results show that our analytical formulation is consistent with state-of-the-art methods in terms of configuration prediction, at the cost of a high number of elements. However, contrary to previous approaches, it has the advantage of providing an analytical formulation of the problem, leading to a straightforward computation of the stability 


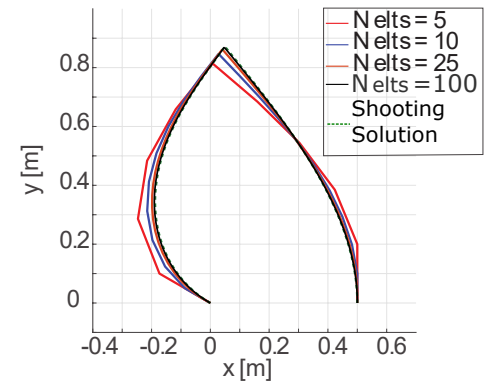

(a) Forward model



(b) Inverse model

Fig. 4. Comparison of our method with respect to the shooting method [5] in terms of robot configuration estimation, as a function of the number of elements $N_{\text {elts }}$ per leg.

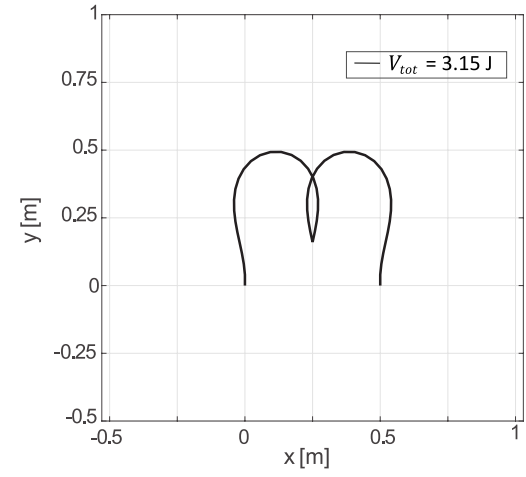

(a) Detected in [2]

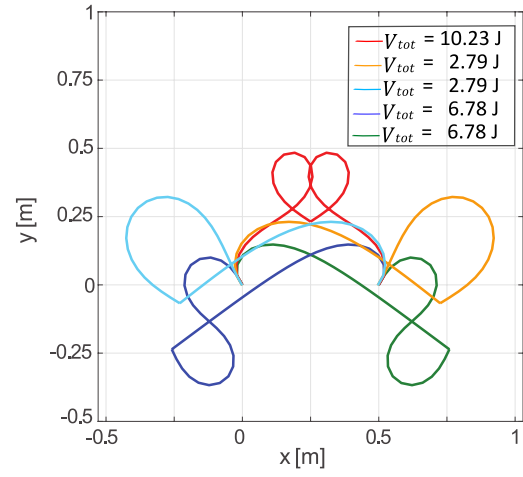

(b) Five unstable configurations found when checking criterion (13), for $q_{a 1}=120 \mathrm{deg}$, $q_{a 2}=60 \mathrm{deg}$

Fig. 5. Unstable configurations.

condition. This formulation can be very useful for the singularity and stability analysis of this type of robots, which is still an open research question.

\section{Conclusion}

In this paper, we provide an analytical formulation of the geometrico-static problem of CPPRs, leading to an analytical computation of set of equations that must be solved in order to find equilibrium configurations. We also provide a stability criterion. This formulation is based on the use of the Kirchhoff's rod theory, and the use of finitedifference approximations. This formulation leads to a quadratic expression of the rod deformation energy.

The solutions provided by our method were compared with the geometrically exact solutions computed with the shooting method. Results show that, for having a good prediction accuracy, a high number of elements must be used. However, the analytical 
formulation of the problem, leading to a straightforward computation of the stability condition, is a major advantage, as it can be very useful for the singularity and stability analysis of CPPRs which is still an open research question. In the future, the complete analysis of the geometrico-static performance of several CPPRs, among which the $\underline{R} F R F \underline{R}$ robot, will be carried out with our method.

\section{References}

1. Altuzarra, O., Caballero, D., Campa, F., Pinto, C.: Forward and inverse kinematics in 2dof planar parallel continuum manipulators. In: Proceedings of the European Conference on Mechanism Science (EuCoMeS 2018). pp. 231-238. Aachen, Germany (2018)

2. Altuzarra, O., Caballero, D., F.J.Campa, Pinto, C.: Position analysis in planar parallel continuum mechanisms. Mechanism and Machine Theory 132, 13-29 (2019)

3. Altuzarra, O., Caballero, D., Zhang, Q., Campa, F.: Kinematic characteristics of parallel continuum mechanisms. In: Proceedings of Advances in Robot Kinematics (ARK 2018). pp. 293-301. Bologna, Italy (2018)

4. Altuzarra, O., Merlet., J.: Certified kinematics solution of 2-dof planar parallel continuum mechanisms. In: Proceedings of the IFToMM World Congress on Mechanism and Machine Science. pp. 197-208. Cracow, Poland (2019)

5. Black, C., Till, J., Rucker, D.: Parallel continuum robots: Modeling, analysis, and actuationbased force sensing. IEEE Transactions on Robotics 34(1), 29-47 (2017)

6. Bryson, C.E., Rucker, D.C.: Toward parallel continuum manipulators. In: 2014 IEEE International Conference on Robotics and Automation (ICRA). pp. 778-785. IEEE (2014)

7. Burgner-Kahrs, J., Rucker, D., Choset, H.: Continuum robots for medical applications: A survey. IEEE Transactions on Robotics 31(6), 1261-1280 (2015)

8. Chikhaoui, M.T., Lilge, S., Kleinschmidt, S., Burgner-Kahrs, J.: Comparison of modeling approaches for a tendon actuated continuum robot with three extensible segments. IEEE Robotics and Automation Letters 4(2), 989-996 (2019)

9. Chirikjian, G.S., Burdick, J.W.: Kinematically optimal hyper-redundant manipulator configurations. IEEE transactions on Robotics and Automation 11(6), 794-806 (1995)

10. Nocedal, J., Wright, S.: Numerical Optimization. Springer, 2nd edn. (2006)

11. Peyron, Q., Rabenorosoa, K., Andreff, N., Renaud, P.: A numerical framework for the stability and cardinality analysis of concentric tube robots: Introduction and application to the follow-the-leader deployment. Mechanism and Machine Theory 132, 176-192 (2019)

12. Till, J., Rucker, D.: Elastic stability of Cosserat rods and parallel continuum robots. IEEE Transactions on Robotics 33(3), 718-733 (2017) 